\title{
Acknowledgement to Reviewers of Journal of Imaging in 2018
}

\author{
Journal of Imaging Editorial Office \\ MDPI, St. Alban-Anlage 66, 4052 Basel, Switzerland \\ Published: 9 January 2019
}

\begin{abstract}
Rigorous peer-review is the corner-stone of high-quality academic publishing. The editorial team greatly appreciates the reviewers who contributed their knowledge and expertise to the journal's editorial process over the past 12 months. In 2018, a total of 142 papers were published in the journal, with a median time to first decision of 20 days and a median time to publication of 57 days. The editors would like to express their sincere gratitude to the following reviewers for their cooperation and dedication in 2018:
\end{abstract}

\author{
Abboud, Marie \\ Abuzaid, Ahmed \\ Achanta, Radhakrishna \\ Addison, Owen \\ Afonso, Manya \\ Agrafiotis, Panagiotis \\ Ahlberg, Jörgen \\ Ahmad, Kashif \\ Alaei, Alireza \\ Alavi, Azadeh \\ Al-Balooshi, Fatema \\ Albu, Felix \\ Ali, Sharib \\ Aliotta, Francesco \\ Al-Machot, Fadi \\ Alparone, Luciano \\ Amerini, Irene \\ Amirshahi, Seyed Ali \\ Amundarain, Aiert \\ Anagnostopoulos, Christos-Nikolaos \\ Ando, Jun \\ Andreasen, Jens Wenzel \\ Anees, Amir \\ Appiah, Kofi \\ Arafin, Md Tanvir \\ Arboleda, Carolina \\ Arnal, Bastien \\ Arrighetti, Walter \\ Atkinson, Jonathan A \\ Aubry, Mathieu \\ Audenaert, Jan \\ Augereau, Olivier \\ Awad, Ali Said
}

\author{
A $\beta$ mann, Marc \\ Bakos, Jason \\ Balázs, Péter \\ Balietti, Stefano \\ Balletti, Caterina \\ Balsa-Barreiro, Jose \\ Banhart, John \\ Banic, Nikola \\ Barney Smith, Elisa \\ Barros, Pablo \\ Barry, David J \\ Barsanti, Sara Gonizzi \\ Baselice, Fabio \\ Bech, Martin \\ Belaid, Abdel \\ Bellani, Marcella \\ Belyaev, Evgeny \\ Berezin, Mikhail Y. \\ Bernabe Garcia, Sergio \\ Berretti, Stefano \\ Berthoumieu, Yannick \\ Bhagat, Yusuf \\ Bilheux, Jean \\ Biliński, Piotr \\ Bilodeau, Michel \\ Birnbacher, Lorenz \\ Blascheck, Tanja \\ Bleay, Steve \\ Bociort, Florian \\ Borzage, Matt \\ Bosilj, Petra \\ Botta, Marco \\ Boyang, Su
}


Brox, Piedad

Bulatov, Dimitri

Burie, Jean-Christophe

Caballer, Jaime Almonacid

Cabrera, Humberto

Calbet, Xavier

Calvo-Zaragoza, Jorge

Campen, Marcel

Campi, Massimiliano

Caplins, Benjamin W.

Cardoso, Tiago

Carminati, Maria Chiara

Carter, William E.

Caruso, Giovanni

Carvajal, Johanna

Castillo, José Maria

Cecotti, Hubert

Cernadas, Eva

Chabiniok, Radomir

Chang, Michael

Chang, Young Ki

Charbonnier, Pierre

Chen, Mingzhou

Chen, Xi

Choi, Woo June

Choi, Wookjin

Chung, Yongwha

Ciuc, Mihai

Ciuciu, Philippe

Clement, Yuen

Coakley, Kevin J.

Coelho, João Pinto

Collins, Christopher M.

Colom, Miguel

Conze, Pierre-Henri

Corchs, Silvia

Cordolino Sobral, Andrews

Corsini, Massimiliano

Cortesi, Marco

Courtney, Jane

Coutrot, Antoine

Cozzolino, Daniel

Da Silva, Julio Cesar

Danescu, Radu

Danilov, Alexander

Davies, Roy

Davila, Kenny

De Aguiar, Hilton B.

De Momi, Elena

De Senneville, Baudouin Denis

Debattista, Kurt

Debeir, Olivier
Delaney, John K.

Delgado, Emma

Dell'Aversano, Angela

Deniziak, Stanisław

Desjardins, Michèle

Dev, Soumyabrata

Diaz, Oliver

Diem, Markus

Diez, Yago

Doitsidis, Lefteris

Dominguez, Enrique

Drukker, Karen

Du, Xinli

El-Hadedy, Mohamed

El-Mashtoly, Samir F.

Elshaw, Mark

Endrizzi, Marco

Engvall, Jan

Fabelo, Himar

Fales, Andrew

Falk, Thorsten

Fanelli, Anna Maria

Fedoseev, Victor

Fernández Carrobles, María Del Milagro

Fernández-Caballero, Antonio

Ferreira, Diogo R.

Fiandrotti, Attilio

Filip, Jiri

Fischer, Gregory

Fisher, Robert

Fliegel, Karel

Florea, Corneliu

Forczmanski, Pawel

Fotopoulos, Vassilis

Frances-Villora, Jose Vicente

Freedman, Jacob

Fryskowska, Anna

Fularz, Michal

Galdran, Adrian

Garnero, Gabriele

Garrido, Mario

Garrod, Oliver G.B.

Gasparini, Francesca

Ge, Yufeng

George, Deepu

Georgopoulos, Andreas

Géraud, Thierry

Gijsenij, Arjan

Ginsca, Alexandru Lucian

Giotis, Angelos P.

Glowacz, Adam

Goltz, Douglas M. 
Gómez, Alberto

González Ruiz, Vicente

Gosak, Marko

Gottfried, Björn

Grant, Gerald T.

Grosse, Mirco

Guerrero, Josechu

Hafner, David

Haji Maghsoudi, Omid

Hallberg, Tomas

Hamada, Shigeru

Hamann, Elias

Hansen, Mark F.

Harada, Kenji

Harguess, Josh

Harjo, Stefanus

Harmsen, Stefan

Hartmann, William

Hast, Anders

Hatt, Mathieu

Hayakawa, Takeshi

Hayakawa, Yoshihiko

Henry, Mary C.

Heras, Dora Blanco

Herkommer, Alois

Hernández, Diego Ortego

Hernandez-Cabronero, Miguel

Herrero-Langreo, Ana

Herrmann, Christian

Hess, Mona

Hines, Andrew

Hiraki, Takao

Hirsch, Ernest

Huang, Xiaojing

Huang, Yanbo

Hudec, Robert

Hui, Jie

Hulusic, Vedad

Hurtík, Petr

Impedovo, Donato

Inagaki, Tetsuya

Inoue, Kohei

Inverso, Donato

Inzerillo, Laura

Janowczyk, Andrew

Javed, Sajid

Jayasuriya, Suren

Jean-Christophe, Prévotet

Jessie, Jockerst

Johnson Shepherd, Jami

Josep, Arnal

Joseph, James
Jung, Ho Gi

Kaestner, Anders

Kaliakatsos-Papakostas, Maximos

Kaplun, Dmitrii

Kardjilov, Nikolay

Kasiński, Krzysztof

Kasneci, Enkelejda

Kastner, Johann

Kawabata, Norifumi

Kawanishi, Yasutomo

Kesidis, Anastasios L.

Keskinarkaus, Anja

Khimchenko, Anna

Kiefer, Gundolf

Kiefer, Johannes

Kim, Chunwoo

Kim, Kyungsang

Kobashi, S

Kober, Silvia

Kok Sheik, Wong

Komoike, Yuta

Konidaris, Thomas

Kraft, Marek

Krähenbühl, Adrien

Krawiec, Krzysztof

Kronreif, Gernot

Krug, Johannes

Kubat, Ivana

Kulhavy, David L.

Kumhálová, Jitka

Kunjachan, Sijumon

Kwok, Edmund

Kwok, Ngai Ming

Laaß, Michael

Laligant, Olivier

LaManna, Jacob

Lambers, Martin

Laneve, Giovanni

Lang, Jochen

Langovoy, Mikhail

Lapray, Pierre-Jean

Leach, Joseph

Lee, Byung-Uk

Lee, Changho

Lee, Jong Taek

Lee, Soochahn

Leeser, Miriam

Leevy, W. Matthew

Lerche, Christoph

Leszczuk, Mikołaj

Li, Gang

Li, Liyuan 
Li, Xiaojiang

Likforman-Sulem, Laurence

LIM, Gilbert

Lin, Yu-Chen

Liu, Jiliang

Liu, Zheng

López, Ainara

Lopez-Molina, Carlos

López-Rubio, Ezequiel

Lorenz, Michael G.

Lucka, Felix

Lui, Yui Man

Luis, Felipe Sesé

Luqman, Muhammad Muzzamil

Lux, Jacques

M. M. Kahaki, Seyed

Maisano, Jessica A.

Majurski, Michael

Mandal, Subhamoy

Martín Fernández, Adrián

Martínez-Hinarejos, Carlos-D.

Materka, Andrzej

Mazurek, Przemysław

Mcllhagga, William

Meiburger, Kristen M.

Meillier, Céline

Mesas-Carrascosa, Francisco Javier

Mestrom, Rob

Miedzińska, Danuta

Miller, Erin

Minervini, Massimo

Moccozet, Laurent

Moghari, Mehdi Hedjazi

Mohammad, Khader

Molinara, Mario

Molina-Solana, Miguel

Mollahosseini, Ali

Mondal, Suman B.

Monnier, Christophe

Moosmann, Julian

Moreno, Emma Muñoz

Morgado, Leonel

Moroni, Davide

Morris, Daniel

Morrison, Melanie

Mortara, Michela

Mothe, Frédéric

Mousas, Christos

Mousavi, Hojjat Seyed

Nakaguchi, Toshiya

Nanni, Loris

Nardino, Vanni
Nasiriavanaki, Mohammadreza

Naumova, Anna V.

Neamtu, Calin

Negru, Mihai

Neilsen, Mitchell L.

Neto, Pedro

Neubert, Jeremiah

Nguyen, Binh P.

Nguyen, Dinh-Liem

Nguyen, Quynh

Nicolas, Stéphane

Nocerino, Erica

Nuutinen, Mikko

Odille, Freddy

Oikawa, Kenichi

Okarma, Krzysztof

Okawa, Shinpei

Olbinado, Margie

Oliveira, João F.

Olsen, Michael

Orlando, José Ignacio

Orović, Irena

O'Shaughnessy, Douglas

Ossandon, Miguel

Oszust, Mariusz

Oyamada, Yuji

Paganin, David

Paige, Rob

Palazzo, Simone

Palmeri, Roberta

Pan, W. David

Panáková, Daniela

Pander, Tomasz

Pandey, Shashank S.

Park, Won-Kwang

Parkinson, Dilworth

Parnell, Steven

Parziale, Antonio

Patella, Marco

Payá, Luis

Pedroni, Marco

Pelzer, Georg

Peng, Yan-Tsung

Persia, Fabio

Petro, Ana Belen

Pevny, Tomas

Peysakhovich, Vsevolod

Pham, Minh-Tan

Pięciak, Tomasz

Pierre, Fabien

Pintus, Ruggero

Poigai Arunachalam, Shivaram 
Polder, Gerrit

Polycarpou, Irene

Pont, Sylvia

Ponti, Cristina

Poovvancheri, Jiju

Pop, Ion Octavian

Porter, Glenn

Posselt, Dorthe

Powers, David

Provazník, Ivo

Psannis, Konstantinos

Psarrou, Alexandra

Putzu, Lorenzo

Qi, Guanqiu

Qian, Wei

Quartulli, Marco

Ragia, Lemonia

Ramos Terrades, Oriol

Randazzo, Andrea

Ranjan, Rajeev

Ranju, Mandal

Rasti, Pejman

Reale, Michael J.

Rebelo, Ana

Reisenhofer, Rafael

Remis, Rob F.

Reu, Phillip L.

Reyes-Aldasoro, Constantino Carlos

Rhodin, Helge

Rhouma, Rhouma

Richter, Thomas A.

Rissolo, Dominique

Ristic, Michael

Ritschel, Tobias

Roberson, Gary

Rodrigues, João

Rosado, Alfredo Muñoz

Rosten, Edward

Rotman, Stanley

Roy, Aditi

Saitoh, Takeshi

Sanchez, Victor

Sandfeld, Stefan

Sankaranarayanan, Aswin C.

Santosh, K.C.

Sato, Hirotaka

Savelonas, Michalis A.

Sawall, Stefan

Scafè, Raffaele

Scapaticci, Rosa

Schaff, Florian

Scharf, Toralf
Schillinger, Burkhard

Schrom-Feiertag, Helmut

Schulz, Georg

Schwabe, Mierk

Schwiegerling, Jim

Selci, Stefano

Sharma, Nabin

Shimada, Atsushi

Shirvaikar, Mukul

Sidike, Paheding

Siepel, Françoise J.

Simone, Gabriele

Simonot, Lionel

Sivasubramanian, Kathyayini

Slattery, Ashley D.

Smilgies, Detlef-M.

Smith, Tim A. D.

Smolka, Bogdan

Smyl, Danny

Sona, Giovanna

Song, Sang-Eun

Sorzano, Carlos Oscar Sanchez

Stäb, Daniel

Stafford-Fraser, Quentin

St-Charles, Pierre-Luc

Sterenborg, Dick

Stock, Stuart

Stoean, Catalin

Stoyanov, Danail

Strzelecki, Michal

$\mathrm{Su}$, Hongbo

Sui, Tan

Sun, In-Cheol

Sundstedt, Veronica

Svendsen, Morten Bo Søndergaard

Ta, Hang

Tabkhi, Hamed

Tahmassebi, Amirhessam

Takayama, Kenshi

Tankyevych, Olena

Tarroni, Giacomo

Tarsitano, Achille

Tarvainen, Tanja

Timlin, Jerilyn A.

Topouzelis, Konstantinos

Tornari, Vivi

Torres Vega, Maria

Toyoura, Masahiro

Treibitz, Tali

Treimer, Wolfgang

Tzimiropoulos, Yorgos

Uchiyama, Hideaki 
Ugail, Hassan

Uhring, Wilfried

Ul Alam, Mohammad Arif

Ullrich, Franziska

Upneja, Rahul

Upputuri, Paul Kumar

Vallez, Noelia

Van De Weijer, Joost

Van Droogenbroeck, Marc

Varga, Tamas

Vegas-Sánchez-Ferrero, Gonzalo

Vélez, José Francisco

Verbeek, Fons J.

Verma, Navin Kumar

Vertan, Constantin

Vesoulis, Zachary A.

Vidal, Vicente

Vila-Comamala, Joan

Villanueva, Manuel Vargas

Vincent, Nicole

Walha, Rim

Wang, Depeng

Wang, Zhantong

Watson, Des

Weber, Jonathan

Weiss, Pierre

Weitkamp, Timm
White, Ryan M.

Wiliem, Arnold

Williams, Jeremiah

Wirz, Raul

$\mathrm{Wu}$, Ziyan

Xiao, Perry

Yeom, Seokwon

Yue-Hei Ng, Joe

Yuen, Peter

Zabalza, Jaime

Zabler, Simon

Zaky, Ahmed

Zambrano, Miller

Zanetti, Massimo

Zanibbi, Richard

Zaslanky, Paul

Zauner, Gerald

Zeni, Nicola

Zerman, Emin

Zhang, Jiashu

Zhang, Jiong

Zhang, Ziming

Zherebtsov, Evgeny

Zin, Thi Thi

Zlokolica, Vladimir

Zuber, Marcus

(C) 2019 by the author. Licensee MDPI, Basel, Switzerland. This article is an open access article distributed under the terms and conditions of the Creative Commons Attribution (CC BY) license (http://creativecommons.org/licenses/by/4.0/). 